\title{
Application of Quadrature Rules for Cauchy-Type Integrals to the Generalized Poincaré-Bertrand Formula
}

\author{
By N. I. Ioakimidis
}

\begin{abstract}
The classical Poincaré-Bertrand transposition formula for the inversion of the order of integration in repeated Cauchy-type integrals is generalized in accordance with a new interpretation of Cauchy-type integrals. Next, the Gauss-Jacobi quadrature rule is applied, in a particular case of the generalized Poincare-Bertrand formula, to both members of this formula and it is proved that this formula still remains valid (after the approximation of the integrals by quadrature sums). Two simple applications of this result, one concerning the convergence of a quadrature rule for repeated Cauchy-type integrals, and the other the numerical solution of singular integral equations, are made. Further generalizations and applications of the present results follow easily.
\end{abstract}

1. Introduction. A classical formula in the theory of Cauchy-type principal value integrals is the Poincaré-Bertrand transposition formula (see, e.g., [5], [17]) for the inversion of the order of integration in repeated integrals:

$$
\begin{aligned}
f_{a}^{b} \frac{1}{x-y} & {\left[f_{a}^{b} \frac{f(x, t)}{t-x} d t\right] d x } \\
& =-\pi^{2} f(y, y)+f_{a}^{b}\left[f_{a}^{b} \frac{f(x, t)}{(x-y)(t-x)} d x\right] d t .
\end{aligned}
$$

In this formula, $f(x, t)$ is a Hölder-continuous function with respect to both its variables, but it may have weak power singularities near the endpoints $a, b$ of the integration interval [16]. The Poincaré-Bertrand formula has been proved useful in many applications of Cauchy-type integrals and the corresponding singular integral equations.

In a recent paper, Lifanov [16] has proved the Poincaré-Bertrand formula and generalized it to arbitrarily many dimensions by using a new approach, namely the approximation of the integrals in this formula by quadrature sums (based on appropriate quadrature rules for Cauchy principal value integrals with equispaced nodes) and the use of the convergence property of these rules to the corresponding integrals.

Here, inspired by the work of Lifanov, we will prove the validity of the PoincaréBertrand formula when the Gauss-Jacobi quadrature rule is used for the approximation of the integrals in both sides of this formula (with the same number of nodes $n$ ).

Received March 10, 1983; revised January 17, 1984 and May 30, 1984.

1980 Mathematics Subject Classification. Primary 65D30, 65D32; Secondary 65R20.

(C)1985 American Mathematical Society $0025-5718 / 85 \$ 1.00+\$ .25$ per page 
We will use a generalized (and most probably new) form of the Poincaré-Bertrand formula, which will be derived in the next section, the classical formula (1) being just a special case of our formula.

Since in several cases Cauchy principal value integrals are approximated by quadrature sums, the present results seem interesting. Two applications, the first one concerning the proof of convergence of quadrature rules for repeated Cauchy principal value integrals and the second one concerning the numerical solution of Cauchy-type singular integral equations, will also be made.

2. The Generalized Poincaré-Bertrand Formula. In a recent paper [11] we have generalized the classical principal value interpretation of Cauchy-type integrals along the integration interval. This generalization led to the introduction of a new class of Cauchy-type integrals, denoted by the symbol ${ }^{(c)} f$. For this class of integrals it was proved that [11]

$$
\text { (c) } f_{a}^{b} \frac{f(t)}{t-x} d t=c f(x)+f_{a}^{b} \frac{f(t)}{t-x} d t .
$$

By taking into account this definition, we easily obtain

$$
\begin{aligned}
(-c) f_{a}^{b} \frac{1}{x-y}\left[{ }^{(c)} f_{a}^{b} \frac{f(x, t)}{t-x} d t\right] d x= & -c^{2} f(y, y)+c \int_{a}^{b} \frac{f(t, t)-f(y, t)}{t-y} d t \\
& +f_{a}^{b} \frac{1}{x-y}\left[f_{a}^{b} \frac{f(x, t)}{t-x} d t\right] d x
\end{aligned}
$$

If we invert the order of integration in the same repeated integral, we find

$$
\begin{aligned}
& f_{a}\left[{ }^{b}{ }^{(-c)} f_{a}^{b} \frac{f(x, t)}{(x-y)(t-x)} d x\right] d t \\
& \quad{ }^{(c)} f_{a}^{b} \frac{1}{t-y}\left[{ }^{(-c)} f_{a}^{b}\left(\frac{1}{x-y}-\frac{1}{x-t}\right) f(x, t) d x\right] d t \\
&=c \int_{a}^{b} \frac{f(t, t)-f(y, t)}{t-y} d t+f_{a}^{b}\left[f_{a}^{b} \frac{f(x, t)}{(x-y)(t-x)} d x\right] d t
\end{aligned}
$$

since

$$
\frac{1}{(x-y)(t-x)}=\frac{1}{t-y}\left(\frac{1}{x-y}-\frac{1}{x-t}\right) .
$$

Finally, by comparing the right-hand sides of (3) and (4) and taking into account the Poincaré-Bertrand formula (1) in its classical form, we obtain the generalized Poincaré-Bertrand formula:

$$
\begin{aligned}
& \text { (-c) } f_{a}^{b} \frac{1}{x-y}\left[{ }^{(c)} f_{a}^{b} \frac{f(x, t)}{t-x} d t\right] d x \\
& \quad=-\left(\pi^{2}+c^{2}\right) f(y, y)+{ }^{(c)} f_{a}^{b}\left[{ }^{(-c)} f_{a}^{b} \frac{f(x, t)}{(x-y)(t-x)} d x\right] d t .
\end{aligned}
$$

Of course, for $c=0$ (6) reduces to (1). As far as we know, the above generalization of the Poincaré-Bertrand formula is new. 
3. Application of Quadrature Rules. In this section, we will prove that the generalized Poincaré-Bertrand formula remains valid (under appropriate conditions) if the integrals in both sides of it are approximated by quadrature sums. Of course, the present results also hold true for the classical Poincaré-Bertrand formula (1), a special case of (6).

Although the present results can be modified to apply to many quadrature rules, we will consider only the Gauss-Jacobi quadrature rule. (A special case of this rule is the Gauss-Chebyshev quadrature rule.) For Cauchy-type principal value integrals, the Gauss-Jacobi quadrature rule was proposed by Chawla and Ramakrishnan [1]. For ordinary integrals this rule has the form

$$
\int_{-1}^{1} w(t) g(t) d t=\sum_{i=1}^{n} A_{i n} g\left(t_{i n}\right)+E_{n},
$$

where $w(t)=(1-t)^{\alpha}(1+t)^{\beta}$ and the $t_{\text {in }}$ are the roots of the Jacobi polynomial $P_{n}^{(\alpha, \beta)}(t)$. We shall also need the rule

$$
\int_{-1}^{1} w^{*}(t) g(t) d t=\sum_{k=1}^{n-1} B_{k n} g\left(x_{k n}\right)+E_{n},
$$

where $w^{*}(t)=[w(t)]^{-1}$ and the $x_{k n}$ are the roots of $P_{n-1}^{(-\alpha,-\beta)}(x)$. In our discussion, the parameters $\alpha$ and $\beta$ are related by the equation [9, Eq. (43)]

$$
\kappa=-(\alpha+\beta)=1, \quad-1<\alpha, \beta<0 .
$$

For Cauchy-type integrals, the quadrature rules (7) and (8) are modified as [1], [7], [11]

$$
\begin{aligned}
\text { (c) } f_{-1}^{1} w(t) \frac{g(t)}{t-y} d t= & \sum_{i=1}^{n} A_{i n} \frac{g\left(t_{i n}\right)}{t_{i n}-y}-\left[\frac{\Pi_{n}^{(\alpha, \beta)}(y)}{P_{n}^{(\alpha, \beta)}(y)}-c w(y)\right] g(y) \\
& +E_{n}, \quad y \neq t_{i n}, i=1(1) n, \\
{ }^{(-c) ~} f_{-1}^{1} w^{*}(t) \frac{g(t)}{t-y} d t= & \sum_{k=1}^{n-1} B_{k n} \frac{g\left(x_{k n}\right)}{x_{k n}-y}-\left[\frac{\Pi_{n-1}^{(-\alpha,-\beta)}(y)}{P_{n-1}^{(-\alpha,-\beta)}(y)}+c w^{*}(y)\right] g(y) \\
& +E_{n}, \quad y \neq x_{k n}, k=1(1)(n-1),
\end{aligned}
$$

where the Jacobi functions of the second kind $\Pi_{m}^{(\gamma, \delta)}(y)$ are defined by

$$
\Pi_{m}^{(\gamma, \delta)}(y)=f_{-1}^{1} w(t) \frac{P_{m}^{(\gamma, \delta)}(t)}{y-t} d t .
$$

Here we are interested in the special value of $c$, denoted by $\hat{c}$, defined by [9, Eq.

$$
\hat{c}=-\pi \cot \pi \alpha=\pi \cot \pi \beta,
$$

where (9) is also taken into account. In this case, since [13, Eq. (2.6)]

$$
\Pi_{n}^{(\alpha, \beta)}(y)=\hat{c} w(y) P_{n}^{(\alpha, \beta)}(y)+\frac{\pi}{2 \sin \pi \alpha} P_{n-1}^{(-\alpha,-\beta)}(y),
$$

(10) takes the simpler form (see also [9, Eq. (20)])

$$
\text { (c) } \begin{aligned}
f_{-1}^{1} w(t) \frac{g(t)}{t-y} d t= & \sum_{i=1}^{n} A_{\text {in }} \frac{g\left(t_{i n}\right)}{t_{i n}-y}-\frac{\pi}{2 \sin \pi \alpha} \frac{P_{n-1}^{(-\alpha,-\beta)}(y)}{P_{n}^{(\alpha, \beta)}(y)} g(y) \\
& +E_{n}, \quad y \neq t_{i n}, i=1(1) n .
\end{aligned}
$$


Similarly, since

$$
\Pi_{n-1}^{(-\alpha,-\beta)}(y)=-\hat{c} w^{*}(y) P_{n-1}^{(-\alpha,-\beta)}(y)-\frac{2 \pi}{\sin \pi \alpha} P_{n}^{(\alpha, \beta)}(y),
$$

(11) takes the simpler form (see also [9, Eq. (31)])

$$
\begin{aligned}
(-\hat{c}) f_{-1}^{1} w^{*}(t) \frac{g(t)}{t-y} d t= & \sum_{k=1}^{n-1} B_{k n} \frac{g\left(x_{k n}\right)}{x_{k n}-y}+\frac{2 \pi}{\sin \pi \alpha} \frac{P_{n}^{(\alpha, \beta)}(y)}{P_{n-1}^{(-\alpha,-\beta)}(y)} g(y) \\
& +E_{n}, \quad y \neq x_{k n}, k=1(1)(n-1) .
\end{aligned}
$$

Now, taking $f(x, t)=w^{*}(x) w(t) g(x, t)$, we can rewrite the generalized Poincaré-Bertrand formula (6) as

$$
\begin{aligned}
& { }^{(-c)} f_{-1}^{1} \frac{w^{*}(x)}{x-y}\left[{ }^{(c)} f_{-1}^{1} \frac{w(t) g(x, t)}{t-x} d t\right] d x \\
& \quad=-\left(\pi^{2}+c^{2}\right) g(y, y)+{ }^{(c)} f_{-1}^{1} w(t)\left[(-c) \int_{-1}^{1} \frac{w^{*}(x) g(x, t)}{(x-y)(t-x)} d x\right] d t
\end{aligned}
$$

$g(x, t)$, of course, being assumed a Hölder-continuous function along $[-1,1]$. By taking into account (14) for $n=0$ and (16) for $n=1$ (together with (12)), that is,

$$
\text { (i) } f_{-1}^{1} \frac{w(t)}{t-y} d t=0, \quad{ }^{(-\hat{c})} f_{-1}^{1} \frac{w^{*}(x)}{x-y} d x=\frac{2 \pi}{\sin \pi \alpha} P_{1}^{(\alpha, \beta)}(y),
$$

as well as (5), we rewrite (18) as

$$
\begin{aligned}
(-\hat{c}) f_{-1}^{1} \frac{w^{*}(x)}{x-y} & {\left[\int_{-1}^{1} w(t) \frac{g(x, t)-g(x, x)}{t-x} d t\right] d x } \\
= & -\left(\pi^{2}+\hat{c}^{2}\right) g(y, y)+{ }^{(\hat{c})} f_{-1}^{1} \frac{w(t)}{t-y} \\
& \times\left\{\int_{-1}^{1} w^{*}(x)\left[\frac{g(x, t)-g(y, t)}{x-y}-\frac{g(x, t)-g(t, t)}{x-t}\right] d x\right. \\
& \left.+\frac{2 \pi}{\sin \pi \alpha}\left[P_{1}^{(\alpha, \beta)}(y) g(y, t)-P_{1}^{(\alpha, \beta)}(t) g(t, t)\right]\right\} d t
\end{aligned}
$$

It can be directly seen that, instead of applying the aforementioned Gauss-Jacobi quadrature rules directly to (18), it is preferable, but equivalent, to apply them to (20), which was proved equivalent to (18). Now, by applying (7) and (17) to the left-hand side $I_{l}$ of $(20)$, we find

$$
\begin{aligned}
I_{l}= & \sum_{k=1}^{n-1} \frac{B_{k n}}{x_{k n}-y}\left[\sum_{i=1}^{n} A_{i n} \frac{g\left(x_{k n}, t_{i n}\right)-g\left(x_{k n}, x_{k n}\right)}{t_{i n}-x_{k n}}\right] \\
& +\frac{2 \pi}{\sin \pi \alpha} \frac{P_{n}^{(\alpha, \beta)}(y)}{P_{n-1}^{(-\alpha,-\beta)}(y)}\left[\sum_{i=1}^{n} A_{i n} \frac{g\left(y, t_{i n}\right)-g(y, y)}{t_{i n}-y}\right]+E_{n} .
\end{aligned}
$$


In a similar way, by applying (8) and (15) to the right-hand side $I_{r}$ of (20), we obtain after some simple calculations

$$
\begin{aligned}
I_{r}= & -\left(\pi^{2}+\hat{c}^{2}\right) g(y, y)+\sum_{i=1}^{n} \frac{A_{i n}}{t_{i n}-y} \\
& \times\left\{\sum_{k=1}^{n-1} B_{k n}\left[\frac{g\left(x_{k n}, t_{i n}\right)-g\left(y, t_{i n}\right)}{x_{k n}-y}-\frac{g\left(x_{k n}, t_{i n}\right)-g\left(t_{i n}, t_{i n}\right)}{x_{k n}-t_{i n}}\right]\right. \\
& \left.\quad+\frac{2 \pi}{\sin \pi \alpha}\left[P_{1}^{(\alpha, \beta)}(y) g\left(y, t_{i n}\right)-P_{1}^{(\alpha, \beta)}\left(t_{i n}\right) g\left(t_{i n}, t_{i n}\right)\right]\right\}+E_{n} .
\end{aligned}
$$

Now let $\hat{I}_{l}=I$, but without the error term and $\hat{I}_{r}=I_{r}$ also without the error term. We will prove that

$$
\hat{I}_{l}=\hat{I}_{r} .
$$

To this end, we apply the quadrature rules (15) and (17) to (19). (These rules are exact in this special case.) Then we obtain

$$
\begin{gathered}
\sum_{i=1}^{n} \frac{A_{i n}}{t_{i n}-y}=\frac{\pi}{2 \sin \pi \alpha} \frac{P_{n-1}^{(-\alpha,-\beta)}(y)}{P_{n}^{(\alpha, \beta)}(y)}, \\
\sum_{k=1}^{n-1} \frac{B_{k n}}{x_{k n}-y}=\frac{2 \pi}{\sin \pi \alpha}\left[P_{1}^{(\alpha, \beta)}(y)-\frac{P_{n}^{(\alpha, \beta)}(y)}{P_{n-1}^{(-\alpha,-\beta)}(y)}\right] .
\end{gathered}
$$

Furthermore, for $y=x_{k n}$ and $t_{i n}$, respectively, we find

$$
\sum_{i=1}^{n} \frac{A_{i n}}{t_{i n}-x_{k n}}=0, \quad \sum_{k=1}^{n-1} \frac{B_{k n}}{x_{k n}-t_{i n}}=\frac{2 \pi}{\sin \pi \alpha} P_{1}^{(\alpha, \beta)}\left(t_{i n}\right) .
$$

By taking into account the definitions of $\hat{I}_{l}$ and $\hat{I}_{r}$, as well as (24) and (25), we observe directly that (23) holds true, provided that the following relation is valid

$$
\frac{2 \pi}{\sin \pi \alpha} \frac{P_{n}^{(\alpha, \beta)}(y)}{P_{n-1}^{(-\alpha,-\beta)}(y)} \sum_{i=1}^{n} \frac{A_{\text {in }}}{t_{i n}-y}=\pi^{2}+\hat{c}^{2} .
$$

But this relation, which (because of (24a)) reduces to

$$
\frac{\pi^{2}}{\sin ^{2} \pi \alpha}=\pi^{2}+\hat{c}^{2}
$$

is valid, as can easily be verified from (13).

Therefore, it was proved that the generalized Poincaré-Bertrand formula (6) remains valid (from the numerical point of view) in the case when the Gauss-Jacobi quadrature formula (equivalently, the Gauss-Chebyshev quadrature formula for (1)) is applicable and it is applied to both members of (6). This was proved in the case when $\kappa=-(\alpha+\beta)=1$, but it can also be proved for $\kappa=0$. Then, $-1<\alpha<0<\beta$ $<1$ or $-1<\beta<0<\alpha<1$ (with $\alpha=-\beta$ or $\kappa=-(\alpha+\beta)=0$ ), but (13) holds still true. Moreover, the same result can be proved for other selections of the quadrature rule (e.g., for the Lobatto-Jacobi quadrature rule). In the next two sections, we will apply this result to two interesting problems. 
4. A Convergence Result in Numerical Integration. For $\alpha=\beta=-\frac{1}{2}$ (whence $\hat{c}=0$ ) and $g(x, t)=h(t)$, independent of $x$, the Poincaré-Bertrand formula (18) takes the form

$$
\begin{aligned}
f_{-1}^{1} \frac{\left(1-x^{2}\right)^{1 / 2}}{x-y} & {\left[f_{-1}^{1} \frac{\left(1-t^{2}\right)^{-1 / 2} h(t)}{t-x} d t\right] d x } \\
& =-\pi^{2} h(y)+\pi \int_{-1}^{1} w(t) h(t) d t
\end{aligned}
$$

because of (5), and

$$
f_{-1}^{1} \frac{\left(1-x^{2}\right)^{1 / 2}}{x-y} d x=-\pi y
$$

a special case of the second of (19). It is well-known that the Gauss-Chebyshev quadrature rule converges for continuous integrands; therefore, it converges for Hölder-continuous integrands $h(t)$ too. This means that if we apply this quadrature rule to the right-hand side of (28), we have convergence for $n \rightarrow \infty$. But we have proved in the previous section that for a particular value of $n$, the same numerical results are obtained if the corresponding forms of a Gauss-Jacobi quadrature rule are applied to both sides of (20). This means that if we apply the Gauss-Chebyshev quadrature rule (with $n$ nodes for the weight function $\left.\left(1-t^{2}\right)^{-1 / 2}\right)$ to the approximation of the inner Cauchy principal value integral and a similar quadrature rule (but with $n-1$ nodes for the weight function $\left(1-x^{2}\right)^{1 / 2}$ ) to the approximation of the outer Cauchy principal value integral in the left-hand side of (28), then we obtain a convergent sequence (as $n \rightarrow \infty$ ) of quadrature rules for this repeated Cauchy principal value integral. (Of course, $h(t)$ has to be assumed Hölder-continuous; otherwise, the inner Cauchy principal value integral is not defined in general.)

The above convergence result is very strange since, as is well known, Gaussian quadrature rules for Cauchy principal value integrals converge for integrands possessing a continuous derivative [4], [12] and not being simply Hölder-continuous functions. For Hölder-continuous functions, convergence was proved by Elliott [2] only subject to very strict assumptions for the Gaussian quadrature rule used and, furthermore, for appropriate subsequences of the positive integers and not simply as $n \rightarrow \infty$ as is the case here. On the other hand, Tsamasphyros and Theocaris claimed that the Gauss-Jacobi quadrature rule for Cauchy principal value integrals converges as $n \rightarrow \infty$ for Hölder-continuous functions (without additional restrictions), but their results are not justified. (More explicitly, the crucial error in the proof of [18] seems to be in the second of inequalities (29), whereas in the proof of [20] it is in the argumentation after Eq. (11), which does not take into account the roots $t_{\text {in }}$ of $P_{n}^{(\alpha, \beta)}(t)$ or, equivalently, the poles of $\Psi_{n}^{(\alpha, \beta)}(t) / P_{n}^{(\alpha, \beta)}(t)$ along the integration interval.)

5. Numerical Solution of Singular Integral Equations. The result proved in Section 3 can also be used in the numerical solution of Cauchy-type singular integral equations in the same way that the Poincare-Bertrand formula can be used in the solution of a dominant Cauchy-type singular integral equation of the first kind (inversion of a Cauchy-type singular integral) [5]. 
Let us consider again (18) with $g(x, t)=h(t)$, independent of $x$. In this case, taking into account the second of (19), and (5), as well as the fact that

$$
P_{1}^{(\alpha, \beta)}(y)=\frac{1}{2}(y+\alpha-\beta), \quad \alpha+\beta=-1,
$$

we find from (18)

$$
\begin{gathered}
{ }^{(-\hat{c})} f_{-1}^{1} \frac{w^{*}(x)}{x-y}\left[{ }^{(\hat{c})} f_{-1}^{1} \frac{w(t) h(t)}{t-x} d t\right] d x+\frac{\pi}{\sin \pi \alpha} \int_{-1}^{1} w(t) h(t) d t \\
=-\left(\pi^{2}+\hat{c}^{2}\right) h(y) .
\end{gathered}
$$

By applying the appropriate Gauss-Jacobi quadrature rules to the left-hand side of (31) for $y=t_{l n}(l=1(1) n)$, we obtain

$$
\begin{array}{r}
\sum_{i=1}^{n} A_{i n}\left[\sum_{k=1}^{n-1} \frac{B_{k n}}{\left(t_{i n}-x_{k n}\right)\left(t_{l n}-x_{k n}\right)}-\frac{\pi}{\sin \pi \alpha}\right] h\left(t_{i n}\right) \\
=\left(\pi^{2}+\hat{c}^{2}\right) h\left(t_{l n}\right), \quad l=1(1) n .
\end{array}
$$

This is the fundamental quadrature formula for the numerical solution of the dominant Cauchy-type singular integral equations of the first or the second kind (but with constant coefficients and index $\kappa=1$ ). For $\alpha=\beta=-\frac{1}{2}$ (whence $\hat{c}=0$ ), (32) reduces to

$$
\sum_{i=1}^{n} A_{i n}\left[\sum_{k=1}^{n-1} \frac{B_{k n}}{\left(t_{i n}-x_{k n}\right)\left(t_{l n}-x_{k n}\right)}+\pi\right] h\left(t_{i n}\right)=\pi^{2} h\left(t_{l n}\right), \quad l=1(1) n .
$$

This equation was proved by the author [14] and was the fundamental equation for the proof of the equivalence of the numerical methods for the solution of a Cauchy-type singular integral equation and the equivalent Fredholm integral equation of the second kind. (The results of [14] were taken into account by Gerasoulis and appeared again in [6].) The general case of the Gauss-Jacobi quadrature rule, leading to (32), was considered in [9] and, independently, in [19], whereas further generalizations of the results of [14] were made by Elliott [3] and by the author [8]-[10], [15]. In any case, these results can be obtained from the developments of this paper as was already explained in sufficient detail in the cases of the GaussChebyshev and the Gauss-Jacobi quadrature rules.

Acknowledgement. The results reported here belong to a research project supported by the National Hellenic Research Foundation. The financial support of this Foundation is gratefully acknowledged.

Chair of Mathematics B'

School of Engineering

University of Patras

P.O. Box 1120

GR-261.10 Patras, Greece

1. M. M. Chawla \& T. R. Ramakrishnan, "Modified Gauss-Jacobi quadrature formulas for the numerical evaluation of Cauchy type singular integrals," BIT, v. 14, 1974, pp. 14-21.

2. D. ElliotT, "On the convergence of Hunter's quadrature rule for Cauchy principal value integrals," $B I T$, v. 19,1979 , pp. 457-462.

3. D. ElliotT, “The classical collocation method for singular integral equations," SIAM J. Numer. Anal., v. 19, 1982, pp. 816-832. 
4. D. Elliotr \& D. F. PAGET, "Gauss type quadrature rules for Cauchy principal value integrals," Math. Comp., v. 33, 1979, pp. 301-309.

5. F. D. Gakhov, Boundary Value Problems, Pergamon Press and Addison-Wesley, Oxford, 1966, pp. 49-53.

6. A. Gerasoulis, "On the existence of approximate solutions for singular integral equations of Cauchy type discretized by Gauss-Chebyshev quadrature formulae," BIT, v. 21, 1981, pp. 377-380.

7. N. I. IOAKIMIDIS, "On the numerical evaluation of singular integrals in interface separation problems,” J. Sound Vibration, v. 69, 1980, pp. 167-173.

8. N. I. IOAKIMIDIS, "On the natural interpolation formula for Cauchy type singular integral equations of the first kind," Computing, v. 26, 1981, pp. 73-77.

9. N. I. IOAKIMIDIS, "On the quadrature methods for the numerical solution of singular integral equations,” J. Comput. Appl. Math., v. 8, 1982, pp. 81-86.

10. N. I. IOAKIMIDIS, "A remark on the application of interpolatory quadrature rules to the numerical solution of singular integral equations," J. Comput. Appl. Math. (To appear.)

11. N. I. IOAKImIDIS, "A new interpretation of Cauchy type singular integrals with an application to singular integral equations,” J. Comput. Appl. Math. (To appear.)

12. N. I. IOAKIMIDIS, "On the uniform convergence of Gaussian quadrature rules for Cauchy principal value integrals and their derivatives," Math. Comp., this issue.

13. N. I. IOAKImIDIS \& P. S. ThEOCARIS, "On the numerical solution of a class of singular integral equations,” J. Math. Phys. Sci., v. 11, 1977, pp. 219-235.

14. N. I. IoAkimidis \& P. S. Theocaris, "A comparison between the direct and the classical numerical methods for the solution of Cauchy type singular integral equations," SIAM J. Numer. Anal., v. 17, 1980, pp. 115-118.

15. N. I. IoAkimidis \& P. S. Theocaris, "A remark on the Lobatto-Chebyshev method for the solution of singular integral equations and the evaluation of stress intensity factors," Serdica, v. 6, 1980, pp. 384-390.

16. I. K. Lifanov, "Quadrature formulas and the Poincaré-Bertrand formula for singular integrals," Siberian Math. J., v. 21, 1980 (1981), pp. 787-797.

17. N. I. MuSKhelishvili, Singular Integral Equations, 2nd English ed., Wolters-Noordhoff, Groningen, 1958 , pp. 56-61.

18. G. J. Tsamasphyros \& P. S. Theocaris, "On the convergence of a Gauss quadrature rule for evaluation of Cauchy type singular integrals," BIT, v. 17, 1977, pp. 458-464.

19. G. TSAMASPHYROS \& P. S. TheOcARIS, "Equivalence and convergence of direct and indirect methods for the numerical solution of singular integral equations," Computing, v. 27, 1981, pp. 71-80.

20. G. TSAmasphyros \& P. S. Theocaris, "On the convergence of some quadrature rules for Cauchy principal-value and finite-part integrals," Computing, v. 31, 1983, pp. 105-114. 